\title{
ON Inputs to the OFF Layer: Bipolar Cells That Break the Stratification Rules of the Retina
}

\author{
Hideo Hoshi, Wei-Li Liu, Stephen C. Massey, and Stephen L. Mills \\ Department of Ophthalmology and Visual Science, University of Texas at Houston, Houston, Texas 77030
}

The vertebrate retina is a distinctly laminar structure. Functionally, the inner plexiform layer, in which bipolar cells synapse onto amacrine and ganglion cells, is subdivided into two sublaminae. Cells that depolarize at light offset ramify in sublamina $a$; those that depolarize at light onset ramify in sublamina $b$. The separation of ON and OFF pathways appears to be a fundamental principle of retinal organization that is reflected throughout the entire visual system. We show three clear exceptions to this rule, in which the axons of calbindin-positive $\mathrm{ON}$ cone bipolar cells make ribbon synapses as they pass through the OFF layers with three separate cell types: (1) dopaminergic amacrine cells, (2) intrinsically photosensitive ganglion cells, and (3) bistratified diving ganglion cells. The postsynaptic location of the AMPA receptor GluR4 at these sites suggests that ON bipolar cells can make functional synapses as their axons pass through the OFF layers of the inner plexiform layer. These findings resolve a long-standing question regarding the anomalous $0 \mathrm{~N}$ inputs to dopaminergic amacrine cells and suggest that certain $\mathrm{ON}$ bipolar cell axons can break the stratification rules of the inner plexiform layer by providing significant synaptic output before their terminal specializations. These outputs are not only to dopaminergic amacrine cells but also to at least two $\mathrm{ON}$ ganglion cell types that have dendrites that arborize in sublamina $a$.

\section{Introduction}

The inner plexiform layer (IPL) of the retina is functionally stratified according to the polarity of bipolar cell inputs (Famiglietti et al., 1977; Wässle, 2004). The response polarity of the 10-12 types of bipolar cell is determined by the differential expression of postsynaptic glutamate receptors, in which OFF cone bipolar cells express AMPA/kainate receptors (DeVries, 2000) and ramify in sublamina $a$ of the IPL, whereas ON bipolar cells express metabotropic glutamate mGluR6 receptors and descend to sublamina $b$ (Nomura et al., 1994). This separation of ON and OFF pathways is a fundamental principle of retinal organization. However, dopaminergic amacrine cells (DACs) do not fit neatly into this mold. Most of their dendrites ramify high in the OFF sublayer and receive bipolar input at ribbon synapses, yet they produce an ON response to light (Zhang et al., 2007).

Several mechanisms have been proposed to overcome this difficulty. For example, the ON inputs to DACs might arise from the minor bands in sublamina $b$, although only the dense band in sublamina $a$ is known to receive bipolar cell input (Hokoc and Mariani, 1987). Additionally, these sparse bands may belong to a second type of catecholaminergic cell (Tauchi et al., 1990). DACs could be driven by the sign-inverting glutamate receptor mGluR6, but these receptors are restricted to

Received Feb. 18, 2009; revised May 22, 2009; accepted May 30, 2009.

This research was supported by National Institutes of Health Grants EY06515 (S.C.M.), EY10121 (S.L.M.), and Vision Core Grant EY10608, and by an unrestricted Award from Research to Prevent Blindness to the Department of Ophthalmology and Visual Science, University of Texas at Houston Health Science Center.

Correspondence should be addressed to Stephen L. Mills, Department of Ophthalmology and Visual Science, University of Texas at Houston, 6431 Fannin, MSB 7.024, Houston, TX 77030. E-mail: stephen.I.mills@uth.tmc.edu. D0I:10.1523/JNEUROSCI.0912-09.2009

Copyright $\odot 2009$ Society for Neuroscience $\quad$ 0270-6474/09/298875-09\$15.00/0
ON bipolar dendrites in the outer plexiform layer. Alternatively, an intermediary GABAergic amacrine cell might provide the sign inversion, but the light responses of DACs are not blocked by GABA antagonists (Zhang et al., 2007). A bistratified bipolar cell could provide ON inputs in both sublaminae (Marshak, 2001), but these have not been reliably found in mammalian retinas. In summary, there is no clear explanation for ON inputs to DACs in sublamina $a$.

Zhang et al. (2008) suggested that melanopsin-containing intrinsically photosensitive ganglion cells (ipRGCs) may provide input to one class of DACs. The spectral signature and sustained features of the sustained type of DAC match those of the ipRGCs. Furthermore, photoreceptor degeneration did not block the sustained DAC response. However, the transient light responses of DACs were abolished by pharmacological blockade of the ON bipolar pathway. Additionally, the light responses of all lightdriven ipRGCs are ON driven, despite the fact that the dendrites stratify predominantly in sublamina $a$ (Hattar et al., 2002; Dacey et al., 2005). Thus, any ipRGC input to DACs provides only a partial explanation, and the inputs to the ipRGCs in sublamina $a$ also break the stratification rules.

Recently, we have characterized an unusual bistratified ganglion cell type whose OFF dendrites return to sublamina $b$ to terminate. This ganglion cell spikes only at light onset and contains dendritic spines in sublamina $a$ that contact ON bipolar cells as their axons descend through the OFF sublamina. Synaptic ribbons and glutamate receptors are also present at these conjunctions. We found that these bipolar cells also make synaptic contacts in sublamina $a$ with DACs and ipRGCs. These novel bipolar cell inputs break the stratification rules for the IPL and may account for the anomalous $\mathrm{ON}$ responses of several postsynaptic targets in the OFF layers of the IPL. 


\section{Materials and Methods}

Adult albino rabbits were deeply anesthetized with injections of urethane $(1.5 \mathrm{~g} / \mathrm{kg}$, i.p.) and then humanely killed by intracardial injection of $5 \mathrm{cc}$ urethane after removal of the eyes. All procedures were in accordance with the guidelines of the University of Texas at Houston Animal Welfare Committee. Some retinas were removed from the sclera and placed on a black membrane filter, ganglion cell side up, inserted into a perfusion chamber (Warner Instruments) and then placed on the stage of an Olympus BX50WI upright epifluorescent microscope. Other retinas were cut into pieces and placed into the perfusion chamber with the sclera attached. The tissue was superfused during the experiment with Ames' medium (Sigma-Aldrich) bubbled with $95 \% \mathrm{O}_{2} / 5 \% \mathrm{CO}_{2}$.

Cell targeting and injection. Ganglion cells were stained by application of a few drops of the nuclear fluorescent stain acridine orange (Invitrogen) dissolved in Ames' medium (Sigma-Aldrich) and applied to the retina as needed to visualize the ganglion cell population. With experience, several ganglion cell classes can be targeted with high efficiency. In this study, these include a novel bistratified ganglion cell to be described in detail elsewhere.

Electrophysiology and intracellular staining. Loose patch recordings were made with $1.2 \mathrm{~mm}$ borosilicate glass pulled to a resistance of 4-6 $\mathrm{M} \Omega$ on a Brown-Flaming horizontal micropipette puller (P-97; Sutter Instruments) and filled with Ames' medium and $0.2 \%$ pyranine (SigmaAldrich). Recordings were made with an NPI EPC-03X amplifier (ALA Scientific) at $1000 \times$ gain and notch filtered at $60 \mathrm{~Hz}$. Data were acquired and analyzed using Experimenter 5.0 (DataWave Technology). Recorded cells were filled with Neurobiotin using a sharp electrode as described below. ON cone bipolar cell inputs were blocked with $100 \mu \mathrm{M} 2$-amino4-phosphono butyrate (L-APB) (Tocris Bioscience). Potential inputs from glycinergic, GABAergic, or cholinergic amacrine cells were blocked with $5 \mu \mathrm{M}$ strychnine (Sigma), $5 \mu \mathrm{M}$ SR95531 [2-(3-carboxypropyl)-3amino-6-(4-methoxyphenyl)pyridazinium bromide] (Tocris Bioscience) or $100 \mu \mathrm{M}$ picrotoxin (Sigma), and $2 \mu \mathrm{M}$ nicotine (Sigma), respectively.

After labeling the ganglion cells with acridine orange, individual ganglion cells were targeted through the epifluorescent microscope with the $40 \times$ water objective and the blue-violet excitation filter $(400-440 \mathrm{~nm})$. Thin-walled glass microelectrodes were tip filled with $0.5 \%$ Lucifer yellow-CH (L-453; Invitrogen) and 3.5\% Neurobiotin (Vector Laboratories) in $50 \mathrm{~mm}$ phosphate buffer and backfilled with $3 \mathrm{~m} \mathrm{LiCl}$. Lucifer yellow was iontophoresed with $1 \mathrm{nA}$ negative current for a few seconds to verify cell penetration and obtain a preliminary identification of the cell type, and then each ganglion cell was filled with Neurobiotin (+1 nA, 3 $\mathrm{Hz}, 10 \mathrm{~min}$ ). After the last cell was injected, the tissue was superfused for at least an additional $30 \mathrm{~min}$ and then fixed in $4 \%$ paraformaldehyde in $0.1 \mathrm{~m}$ PBS, $\mathrm{pH} 7.4$, for $1 \mathrm{~h}$ at room temperature. Cells were visualized by overnight incubation in 1:1000 streptavidin-cyanine 3 (Cy3) (Jackson ImmunoResearch). Tissue was mounted in $50 \% 0.2 \mathrm{M}$ PBS plus $50 \%$ glycerol plus $0.1 \%$ para-phenylenediamine (Sigma) to retard fading by fluorescent illumination.

Micrographs were constructed from TIF files exported from the Carl Zeiss LSM 510 software into Adobe Photoshop (Adobe Systems). Adjustments were made to stretch the histogram of pixel intensities to fill the entire 8 bit range.

Immunohistochemistry. For triple labeling, some retinas were blocked with $3 \%$ donkey serum (Jackson ImmunoResearch) $/ 0.1 \%$ sodium azide/ $0.1 \mathrm{M}$ PBS for $90 \mathrm{~min}$ at room temperature and incubated in primary antibodies with $0.1 \mathrm{M}$ PBS/0.1\% sodium azide/0.3\%Triton X-100 for $7 \mathrm{~d}$ at $4^{\circ} \mathrm{C}$. Then, tissues were rinsed several times in $0.1 \mathrm{M}$ PBS and reincubated in the secondary antibody overnight at $4^{\circ} \mathrm{C}$. Donkey anti-mouse Cy3 and donkey anti-goat Cy5 (1:200; Jackson ImmunoResearch) and donkey anti-rabbit Alexa488 (1:200; Invitrogen) were used as secondary antibodies.

A goat polyclonal antibody to choline acetyltransferase (ChAT) (1: 100; Millipore) was used as a guide in establishing depth of stratification. Synaptic ribbons were labeled with a monoclonal RIBEYE antibody (BD Biosciences Transduction Laboratories CtBP2; used at 1:500). A rabbit polyclonal antibody to calbindin-28 kDa (1:5000; Swant) labeled an ON cone bipolar cell type. DACs were labeled with a sheep antibody to tyrosine hydroxylase (TOH) (1:500; Sigma-Aldrich). Evidence for specificity of these antibodies was previously described (Hoshi and Mills, 2009). An antibody to GluR4 (1:100; Millipore) was used to localized AMPA receptors; when this antibody was used, fixation was for only $15 \mathrm{~min}$ in $4 \%$ paraformaldehyde.

We stained the ipRGCs of the rabbit retina with a commercial antibody to connexin 45 (Cx45) (MAB3101; Millipore), which fortuitously stains the ipRGCs in rabbit retina. Because the epitope and specificity for this commercial Cx45 antibody is uncertain, we will refer to this antibody as the ipRGC antibody to avoid confusion. This antibody does not stain $\mathrm{Cx} 45$ puncta in the rabbit retina but only the ipRGC population. Additional support that this antibody labels ipRGCs was obtained by comparison with a second antibody raised to an $\mathrm{N}$-terminus region of rabbit melanopsin. The melanopsin antibody and the Cx45 antibody were colocalized in the same population of ganglion cells.

Confocal microscopy and quantification of contacts. Fluorescent images of stained material were acquired using a Carl Zeiss LSM 510 confocal microscope. Images were scanned as $0.3 \mu \mathrm{m}$ optical sections; stacks of these images were constructed as needed and specified in the figures. Intensity adjustments were made in Adobe Photoshop (Adobe Systems).

The extent to which apparent contacts occurred between calbindinpositive bipolar cells and second-order neurons was assessed by examining $0.3 \mu \mathrm{m}$ optical sections in whole-mount or vibratome section. Single optical sections were examined rather than projections to avoid false positives, and rotation about the axes of the image was used to verify contact within the limits of light microscopy.

\section{Results}

\section{Calbindin-positive bipolar cells contain axonal synaptic ribbons in sublamina $a$}

Antibodies to calbindin-28 $\mathrm{kDa}$ [calcium binding protein $(\mathrm{CaBP})]$ specifically label a single type of $\mathrm{ON}$ cone bipolar cell in the rabbit retina, as well as horizontal cells and an amacrine cell (Massey and Mills, 1996). If the IPL is subdivided into five strata of equal thickness and $0 \%$ represents the border of the inner nuclear layer (INL) and IPL, then the calbindin-positive bipolar cell ramifies in stratum $4 / 5$, at $\sim 80-85 \%$ depth (Fig. $1 a, d, e$ ), always below the ChAT band in sublamina $b$ and just distal to the rod bipolar cells, which occupy stratum 5 (Fig. $1 a$, green). The calbindin-positive bipolar cell axon terminals are narrowly stratified in sublamina $b$ and contain numerous synaptic ribbons (Fig. $1 e)$.

Using an antibody to RIBEYE, we found that $\sim 90 \%$ of the axons of the calbindin-positive bipolar cell also contain ribbons within their descending axons. These ribbons appear high in sublamina $a$ (Fig. 1b,c) and are wholly contained within the bipolar cell axon. These findings raise the possibility that these axonal ribbons make functional $\mathrm{ON}$ synapses with targets in sublamina $a$ in passing (en passant). Occasionally, small spines or protrusions containing ribbons appear on the bipolar cell axon in stratum 1 (Fig. 1b,c). Rarely $(<1 \%)$, a calbindin-positive bipolar cell axon will branch in sublamina $a$ to produce a very fine process that runs $>5 \mu \mathrm{m}$ (Fig. $1 c$, right arrow).

Calbindin-positive amacrine cells, which contain only conventional synapses, typically contain a single stout dendrite (Fig. $1 d$, arrow) that descends, often diagonally, to the middle of the IPL, in which it branches to form a broad plexus. Careful examination of three-dimensional reconstructions reveals that no ribbons are contained within these descending processes. Because stacked images provide potential for errors should they contain some RIBEYE immunoreactivity apparently on the processes of the amacrine cell, we rotated the images and used single optical sections. This invariably revealed that no ribbons were in fact contained within the amacrine cell process. Supplemental Figure 

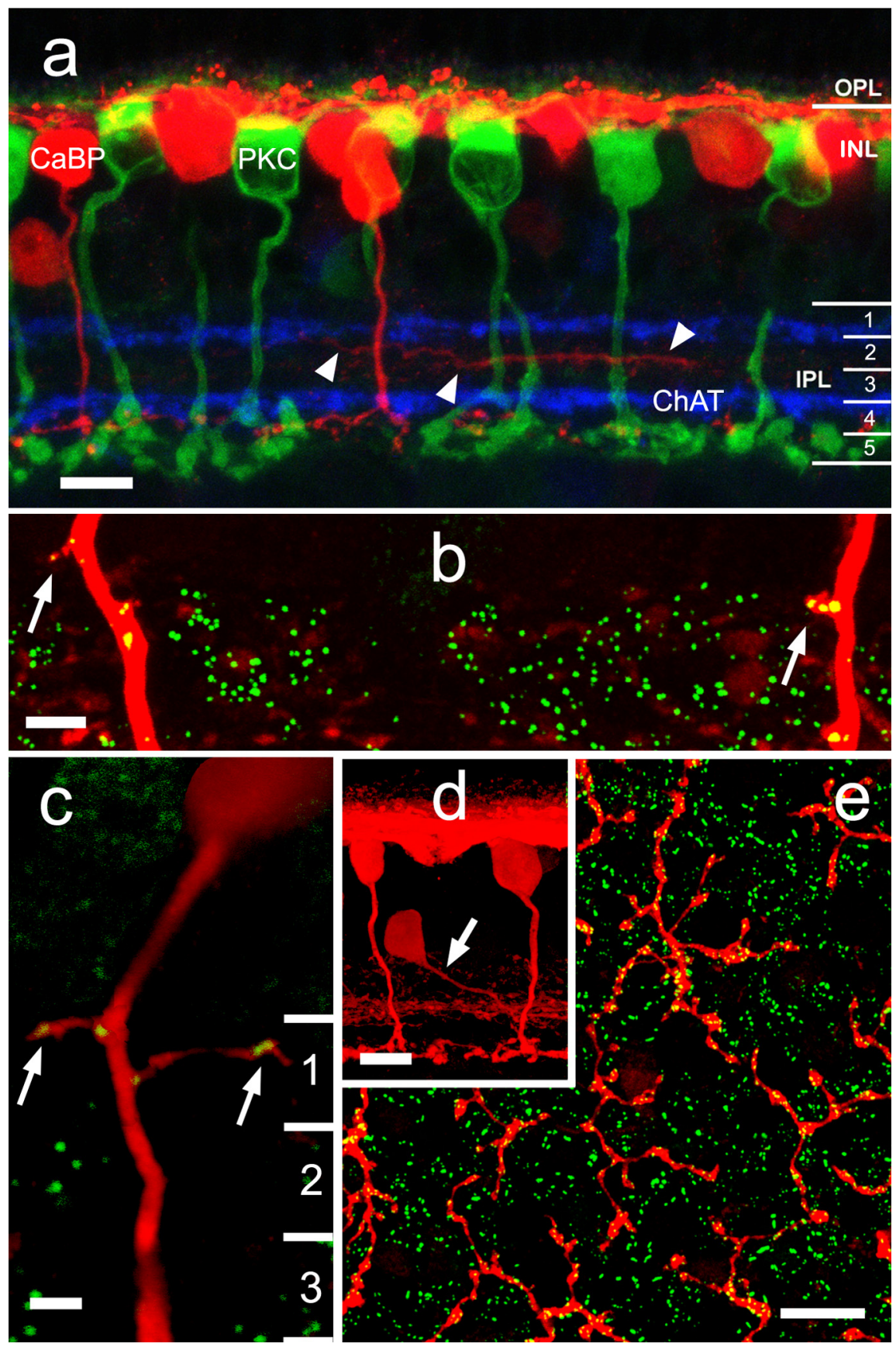

Figure 1. a, An antibody to calbindin-28 kDa (red) stains horizontal cells, an amacrine cell type, and a single population of cone bipolar cell that ramifies in stratum 4 just above the rod bipolar cell end feet (stained by anti-protein kinase $(\alpha$; green). The processes of cholinergic amacrine cells (blue) were stained by anti-choline acetyltransferase. Some of the lateral processes of the amacrine cell ramify below the ChAT band in sublamina $a$ (arrowheads). $\boldsymbol{b}$, Double labeling with an antibody to RIBEYE reveals the presence of synaptic ribbons within the axons themselves or on minor protrusions from them that occur in stratum 1 of sublamina a. c, RIBEYE puncta are occasionally found in rare branches from the axon in stratum 1 of sublamina $\boldsymbol{a}$. $\boldsymbol{d}$, Calbindin-positive amacrine cells give off a single stout process (arrow) that typically descends at an angle to the middle of the IPL before branching. $\boldsymbol{e}$, The majority of ribbons in calbindin-positive bipolar cells are conventionally placed on the axon terminals in sublamina $b$. OPL, Outer plexiform layer. Scale bars: $\boldsymbol{a}, \boldsymbol{d}, 10 \mu \mathrm{m} ; \boldsymbol{b}, \boldsymbol{c}, 2 \mu \mathrm{m} ; \boldsymbol{e}, 25 \mu \mathrm{m}$. Numbers of optical sections of $0.3 \mu \mathrm{m}$ thickness are as follows: $\boldsymbol{a}, \boldsymbol{d}, 5 ; \boldsymbol{b}, \boldsymbol{c}, 1 ; \boldsymbol{e}, \boldsymbol{6}$.

1 (available at www.jneurosci.org as supplemental material) is a rotating three-dimensional reconstruction of calbindin and RIBEYE immunoreactivity that illustrates the relationship between these markers. This figure demonstrates that the calbindinpositive bipolar cell axons often contain giant puncta, usually near the border of the IPL with the INL.
Axonal ribbons from calbindin-positive bipolar cells in sublamina $a$ provide major excitatory input to DACs

All mammalian retinas contain cells that release the neuromodulator dopamine (Witkovsky, 2004). These cells (DACs) can be stained with an antibody to $\mathrm{TOH}$ and may comprise three different subclasses according to differing physiological responses (Zhang et al., 2007, 2008). There is currently no evidence for distinct anatomical classes. Light-driven dopamine release appears to be driven by ON bipolar cells because release is blocked by L-APB (Boelen et al., 1998), but the source of the excitatory drive has never been established. We examined whether the calbindinpositive bipolar cell might make axonal synapses with DACs as well as with ipRGC and bistratified diving ganglion cells.

Six pieces from four rabbit retinas were stained with antibodies to $\mathrm{TOH}, \mathrm{CaBP}$, and RIBEYE. The descending axons of calbindin-positive bipolar cells made frequent contact with $\mathrm{TOH}$-immunoreactive dendrites (Fig. 2a). All DACs contained many hits on major dendrites from these descending axons. Furthermore, synaptic ribbons in the axons as shown by RIBEYE immunoreactivity were reliably found at these sites (Figs. 2b, 3a,b), suggesting that these sites are not chance conjunctions but functional synapses. Close examination of the 10 axons in Figure $2 a$ demonstrated that 8 of 10 both contacted the $\mathrm{TOH}$-stained amacrine cell and contained synaptic ribbons at each site. Of the remaining two axons, one was RIBEYE positive but did not contact the stained DAC, whereas the other appeared to contact the amacrine cell but had no RIBEYE staining at that site.

Indeed, the bipolar cell axons, synaptic ribbons, AMPA receptors, and DAC processes have been found to be colocalized in all possible groups of three markers. The AMPA-receptor subunit GluR4 was invariably found at conjunctions of RIBEYE and DAC processes (Fig. 3c), and GluR4 was found on all DAC processes apposed to calbindin-positive bipolar cell axons (supplemental Fig. 1d, available at www. jneurosci.org as supplemental material). Although this does not indicate that all ribbon synapses onto DACs are from calbindinpositive bipolar cells, it does indicate that all ribbon synapses made by calbindin-positive bipolar cells adjacent to DACs occur in apposition to GluR4 at the amacrine cell postsynaptic density. All of the major components of a functional synapse can be found at these novel contacts with descending bipolar cell axons. This is strong evidence that these sites are functional synapses.

We measured the size and density of RIBEYE puncta in the IPL (Tables 1,2) and found that $88 \%$ of all calbindin-positive bipolar cell 
axons contained RIBEYE labeling in stratum 1. However, these putative ribbons contained in the axons of the calbindin-positive bipolar cell often appeared to be larger than the majority of labeled ribbons not contained in these axons, and it also appeared that these large ribbons especially occurred in association with DAC processes. We quantified this by measuring the size of RIBEYE puncta at 21 locations from 0 to $10 \mathrm{~mm}$ inferior to the visual streak and throughout the 5 strata. We defined giant puncta as having a diameter $>0.8 \mu \mathrm{m}$. Most (88\%) of the giant puncta in stratum 1 were associated with DACs. However, many giant ribbons $(44 \%)$ are not contained in calbindinpositive bipolar cells, suggesting that other ON bipolar cell types may provide en passant inputs as they pass through the DAC matrix in stratum 1. It appears, however, that the calbindin-positive bipolar cell axons constitute a larger fraction of input to the DACs than would be predicted from independent sampling, because calbindinpositive bipolar cells account for approximately one-third of total ON bipolar cell axons (Massey and Mills, 1996) but contain $56 \%$ of the giant puncta.

\section{Some ribbons are located in the INL}

Close examination of the locations of RIBEYE and CaBP double labeling sometimes revealed RIBEYE puncta in axons as they descend through the somas of the inner nuclear layer. Figure $3 d-f$ each show a single $0.3 \mu \mathrm{m}$ optical section from a series taken in the inner nuclear layer. They show that, on some occasions, ribbons can be found directly on the somas of DACs. Figure $6 d$ shows a synaptic ribbon located between the somas of a calbindin-positive bipolar cell and a TOHpositive amacrine cell. This same bipolar cell made another synapse onto the top amacrine cell soma as the axon emerged from the bipolar cell soma (Fig. $3 e$ ) and a third lower on the amacrine cell soma (Fig. $3 f$ ). There is some precedent for somato-somatic synapses in the cat retina (Fisher, 1972), although those were conventional synapses from amacrine cell to bipolar cells. We do not suggest that somato-somatic synapses are a regularly occurring feature of the retina but rather are extreme examples of en passant synapses that occur when the bipolar cell axon contacts a DAC.

Axonal ribbons from calbindin-positive bipolar cells synapse onto intrinsically photosensitive ganglion cells in sublamina $a$

ipRGCs are ganglion cells that have been found in mouse (Berson et al., 2002) and primates (Dacey et al., 2005) that can be

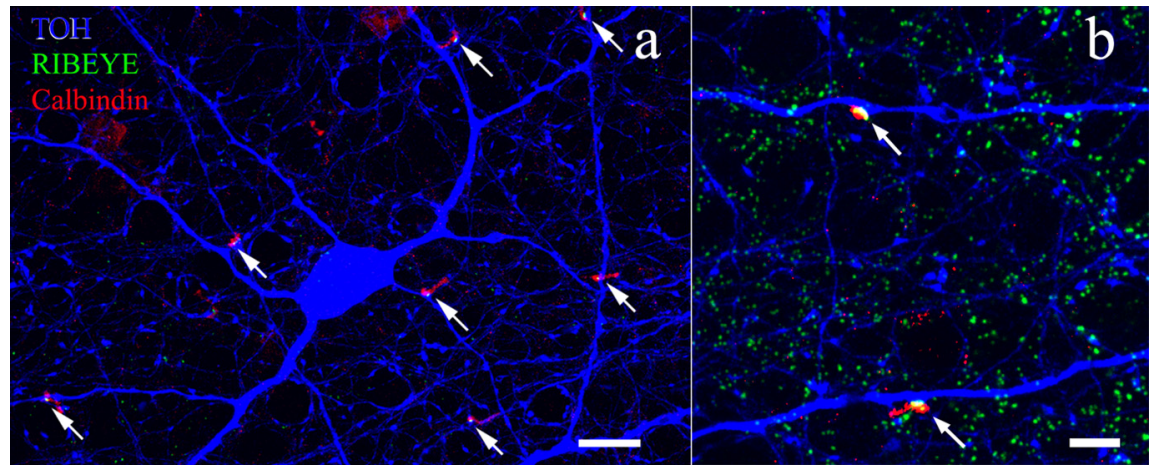

Figure 2. DACs receive excitatory synaptic input from $0 \mathrm{~N}$ cone bipolar cells. $\boldsymbol{a}$, The extensive arbor of the DAC in sublamina $a$ (blue) is revealed by an antibody to TOH. The descending axons of calbindin-positive bipolar cells (red) frequently appear to contact the major dendrites of each amacrine cell. $\boldsymbol{b}$, The conjunctions of DAC processes (blue) with calbindin-positive bipolar cell axons (red) occur in stratum 1 and are also the sites of synaptic ribbons (green; anti-RIBEYE). Scale bars: $\boldsymbol{a}, 20 \mu \mathrm{m} ; \boldsymbol{b}, 5 \mu \mathrm{m}$. Numbers of optical sections of $0.3 \mu \mathrm{m}$ thickness are as follows: $\boldsymbol{a}, 3 ; \boldsymbol{b}, 1$.
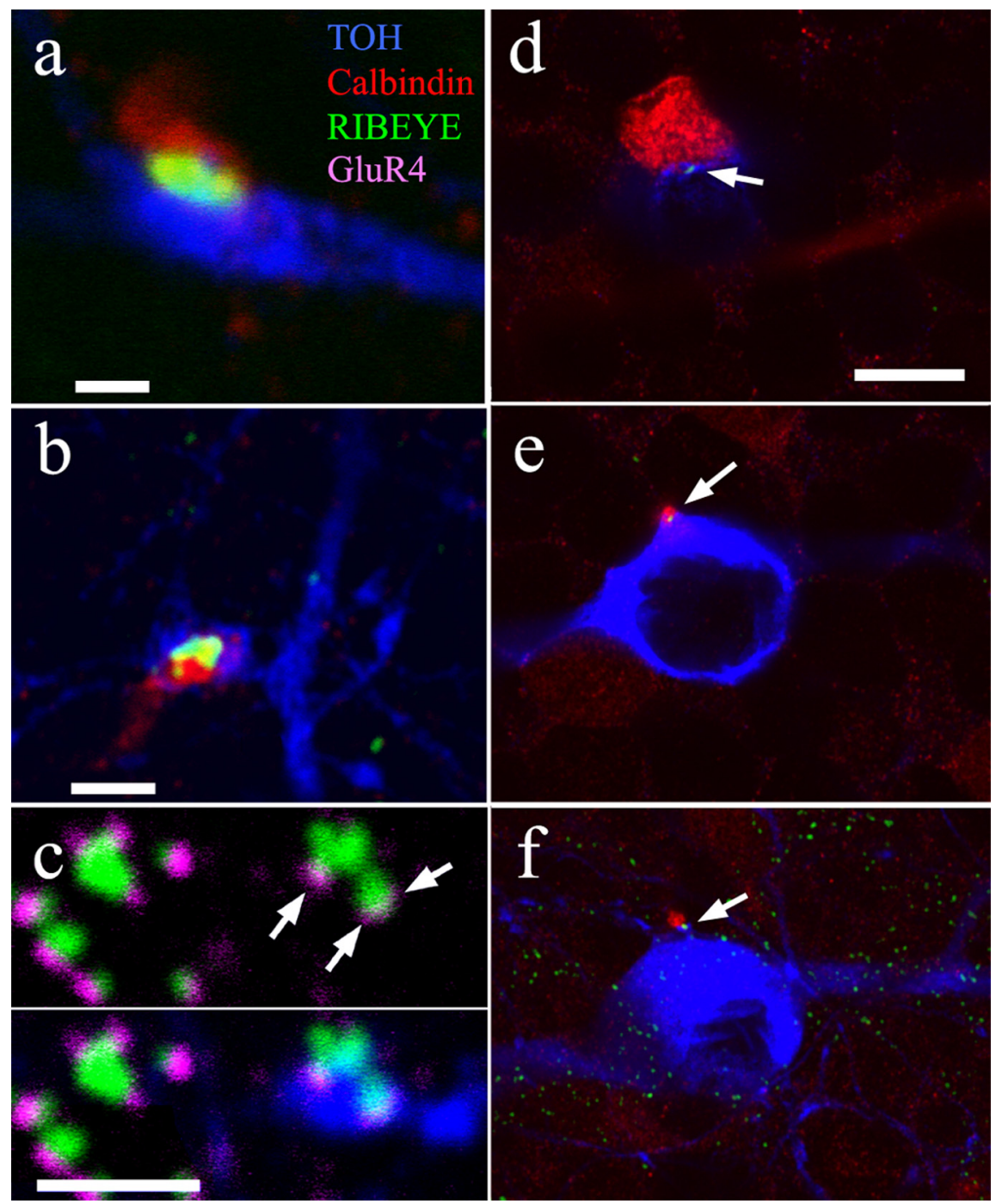

Figure 3. $\quad \boldsymbol{a}, \boldsymbol{b}$, Synaptic ribbons (green) are contained in descending axons of calbindin-positive bipolar cells (red) that contact TOH-positive DACs (blue). c, Ribbon synapses (green) in stratum 1 of sublamina a occur in apposition to the AMPA receptor marker anti-GluR4 (magenta). The bottom of c shows that those ribbons (arrows) that appose TOH-positive cells (blue) also contain the GluR4 marker. Ribbons not on TOH-positive cells also appose GluR4-positive sites. $\boldsymbol{d}$-f, Synaptic ribbons sometimes occur (arrows) on the somas of DACs (blue). These ribbons can occur at abutments of the somas of the amacrine cells with a calbindin-positive bipolar cell (red) (d), as the axon of the bipolar cell emanates from its soma and contacts the upper portion of the amacrine cell (e), or as it contacts a lower portion of the soma again before entering the IPL (f). Scale bars: $\boldsymbol{a}, 1 \mu \mathrm{m} ; \boldsymbol{b}, \boldsymbol{c}, 2 \mu \mathrm{m} ; \boldsymbol{d}-\boldsymbol{f}, 10 \mu \mathrm{m}$. All panels consist of single $0.3 \mu \mathrm{m}$ optical sections. 
Table 1. The quantitative relationship between CaBP-positive axons and RIBEYEpositive puncta of sizes $>0.8 \mu \mathrm{m}$ or smaller

\begin{tabular}{ll}
\hline CaBP bipolar cell axons (21 locations; 4 retinas) & \% (stratum 1 only) \\
\hline \% CaBP axons containing RIBEYE & $87.8 \pm 3.7 \%$ (432 of 492) \\
\% CaBP axons containing giant RIBEYE puncta & $75.6 \pm 11.6 \%$ (372 of 492) \\
\% giant RIBEYE puncta contained in CaBP axons & $56.1 \pm 10.4 \%$ (372 of 663)
\end{tabular}

Percentages are listed as means \pm 1 SD. The number of locations refers to distinct sites from different retinas or different eccentricities that were sampled.

Table 2. The percentage of CaBP-positive axons that contact DACs, ipRGCs, and bistratified diving ganglion cells

\begin{tabular}{lc}
\hline Target cell $(n)$ & \% CaBP axons $\rightarrow$ target cells \\
\hline DAC (21 locations, 4 retinas) & $75.4 \pm 3.3 \%$ (371 of 492) \\
ipRGC (5 locations, 4 retinas) & $6.2 \pm 0.7 \%$ (72 of 1160) \\
Bistratified diving cell (4 locations, 3 retinas) & $18.4 \pm 1.3 \%$ (18 of 98) \\
\hline
\end{tabular}

Percentages are listed as means $\pm 1 S D$. The number of locations refers to distinct sites from different retinas or different eccentricities that were sampled.

driven by either light-driven bipolar cell inputs or by a melanopsin cascade intrinsic to the ganglion cell (Wong et al., 2007; Güler et al., 2008). These cells appear to comprise three different types that can stratify in sublamina $a$, sublamina $b$, or stratify narrowly in both sublaminae (Viney et al., 2007; Baver et al., 2008). All of these ganglion cells appear to depolarize to light onset regardless of their soma location or stratification depth; hence, they are all ON type ganglion cells, even if anomalously stratified in sublamina $a$.

We stained ipRGCs in rabbit retina with a commercial antibody (MAB3101; Millipore) (see Materials and Methods). These cells had the characteristic ipRGC morphology (Fig. 4a), with sparse dendrites that terminate on the dendritic tips of neighboring ipRGCs. Stained ipRGCs were examined for contacts with calbindin-positive bipolar cells in sublamina $a$ as well as the presence of synaptic ribbons using the RIBEYE antibody. Several samples across the retina indicated that calbindin-positive bipolar cell axons often contacted dendrites of ipRGCs in sublamina $a$ (Fig. 4b). These contacts were scattered throughout the dendritic tree of ipRGCs. The axonal arbors of calbindin-positive bipolar cells also regularly contacted the arbors of ipRGCs in sublamina $b$ (Fig. 4c). Approximately $6 \%$ of the descending calbindin-positive axons made contacts with ipRGCs in sublamina $a$ when examining large $\left(\sim 1 \mathrm{~mm}^{2}\right)$ patches of the retina (Tables 1,2). Given the sparseness of the dendritic fields of the ipRGCs, the low percentage of contacts is not surprising. Indeed, if counts are made on $0.01 \mathrm{~mm}^{2}$ patches centered on a soma, in which the density of processes is higher, the percentage of axonal synapse to ipRGC contacts rises to $\sim 13 \%$. It may also indicate that contacts are made preferentially close to the somas, perhaps maximizing their influence. It should be emphasized that, despite the low percentage of axons making contact with ipRGCs, 100\% of the ipRGCs received such contacts in stratum 1 , as with the DACs. These sites were also immunopositive for RIBEYE, as shown for sublamina $a$ in Figure $4 d$ and for GluR4 (supplemental Fig. $2 a-c$, available at www.jneurosci.org as supplemental material), strongly suggesting the presence of functional synapses.

\section{Axonal ribbons from calbindin-positive bipolar cells regularly synapse on a bistratified ganglion cell type in sublamina $a$}

We have recently to learned target and stain $(n=76)$ a specific type of bistratified ganglion cell that can be easily distinguished by size, stratification depth, branching pattern, morphology, and physiology from the other bistratified ganglion cells in the rabbit retina, such as the partly bistratified G3 ganglion cell (Hoshi and Mills, 2009) and the ON-OFF directionally selective ganglion cell (Amthor et al., 1984). We call this cell, which does not appear in the most comprehensive catalog of rabbit ganglion cells (Rockhill et al., 2002), the bistratified diving ganglion cell. This is attributable to the observation that the dendrites that branch in sublamina $a$ do not terminate there but abruptly descend back to stratum $4 / 5$ (Fig. $5 a, b$ ). They terminate there at the same depth as the ON arbor of the cell but farther from the soma than the ON dendrites that remain exclusively in sublamina $b$. The clearest diagnostic feature is that some dendrites that ramify in sublamina $b$ initially appear isolated but can be traced back to the somas by following them first up to the arbor in sublamina $a$ and then back to the soma. In Figure $5 a$, which is color coded by depth, several of these transitions from the OFF layer (cyan) back to the ON layer (red) are marked by arrows. A $z$-rotation (Fig. 5, inset) shows one such transition.

The bistratified diving ganglion cell may be the same as a cell recorded previously by Roska and Werblin (2003) called the type 9 cell. Spiking is blocked in these cells by application of the mGluR6 agonist L-APB (supplemental Fig. 3, available at www. jneurosci.org as supplemental material) (nine of nine cells) (Roska et al., 2006), indicating a lack of OFF cone bipolar cell input to the OFF arbor of this ganglion cell type. An alternative interpretation is that the ganglion cell spikes at light onset are attributable to disinhibition of an amacrine cell ultimately driven by ON bipolar cells. However, because the sign reversal of the inhibitory amacrine cell would lead to depolarization at light offset, a chain of amacrine cells would be required. To test such a possibility, light activity was recorded in the presence of the glycine-receptor antagonist strychnine ( $5 \mu \mathrm{M}$; six of six cells) or the GABA-receptor antagonists SR95531 ( $5 \mu \mathrm{M}$; 12 cells) or picrotoxin (100 $\mu \mathrm{M}$; three cells) and nicotine ( $2 \mu \mathrm{M}$; four cells). In each case (data not shown), spiking was enhanced rather than abolished, eliminating an obligatory role of traditional amacrine cells in initiating spiking. The only remaining amacrine cell pathway would be a potential glutamatergic amacrine cell as proposed by Pickard et al. (2009). These cells have been reported in the retina of rodents (Johnson et al., 2004) but not rabbit (Marc and Jones, 2002). However, the stratification patterns in the rodent are not appropriately placed to account for the synaptic contacts reported here.

We examined small dendritic spines, which are likely to be postsynaptic specializations, on four bistratified diving ganglion cells for apposition to presynaptic markers. We found that these spines were often adjacent to descending axons of the calbindinpositive bipolar cell (Fig. 6a). Close examination of these sites (Fig. 6a1-a5) invariably revealed additional immunolabeling for RIBEYE within the axons and adjacent to the dendritic spines. Examination at high power confirmed that RIBEYE contained in the calbindin-positive bipolar cell axon was closely apposed to the spines of the bistratified diving ganglion cell (Fig. 6b), as if the function of a ganglion cell spine were to reach out to a nearby bipolar axon.

We obtained additional evidence that these points of contact between the descending bipolar cell axons and the spines of the bistratified diving ganglion cell were actual sites of synaptic contact by incubating some pieces of tissue containing the Neurobiotin-injected ganglion cells with an antibody to the AMPA receptor subunit GluR4. Figure $6 c$ demonstrates that GluR4-immunopositive puncta (magenta) are located on spines of the bistratified diving ganglion cell (cyan) and apposed to 
synaptic ribbons (green). Thus, we found an ON bipolar cell axon that contains synaptic ribbons high in sublamina $a$ and that is apposed to a postsynaptic glutamate receptor located on the spines of an ON-type ganglion cell that is bistratified in sublamina $a$. The presence of both presynaptic and postsynaptic markers strongly suggests that these are functional synapses. As with the previous target cells, $100 \%$ of the bistratified diving ganglion cells received inputs from the axons of calbindinpositive bipolar cells in stratum 1 .

Systematic analysis of the bistratified diving ganglion cell in Figures 5 and 6, which was also stained for $\mathrm{CaBP}$ and RIBEYE, showed that the ganglion cell contained 62 dendritic spines in sublamina $a$, of which 57 (92\%) were apposed by ribbons, and that 18 of the $62(29 \%)$ spines were adjacent to calbindin-positive bipolar cell axons, all 18 of which also contained RIBEYE at these sites. As with the DACs, the spines not adjacent to calbindin-positive bipolar cell axons may be sites of similar contact by axons of additional ON cone bipolar cell types.

The number of calbindin-positive bipolar cells contained within the OFF arbor of this bistratified diving ganglion cell that made apparent synaptic contact with its spines was 18 of 98 (18.4\%; mean of four cells) (Tables 1,2). Of course, the number of synaptic ribbons in the axons of calbindin-positive bipolar cells as they descend through sublamina $a$ is small compared with those in the extensive axonal arbors, numbering only $1.5 \%$ of all ribbons in a sample of three neighboring bipolar cells. It is clear that the bulk of excitatory input onto the bistratified diving ganglion cell occurs in sublamina $b$. The smaller but regular occurrence of synapses in sublamina $a$ may serve a specialized purpose.

\section{Axonal ribbons of calbindin-positive bipolar cells do not contact other ganglion cells in sublamina $a$}

We also examined the descending axons of calbindin-positive bipolar cells for synaptic contacts with other ganglion cells that are bistratified in sublamina $a$. These include the $\mathrm{G} 3$ ganglion cell (Hoshi and Mills, 2009), whose major arbor is above the ChAT band in sublamina $a$, and also the ON-OFF directionally selective ganglion cell, whose ON and OFF arbors ramify within the ChAT bands. We were unable to find regular appositions between the descending axons and the dendrites of either cell, or appositions with dendrites of ganglion cells that ramify exclusively in sublamina $a$, such as the OFF $\alpha$ ganglion (G11a) and OFF $\delta$ (G9) ganglion cells. When the processes were close by chance, specializations such as spines or varicosities were not found on the ganglion cells, and in no cases were RIBEYE puncta found in the descending axons at these locations. The absence of presynaptic and postsynaptic markers suggests that the axonal synapses made by calbindin-positive bipolar cell in sublamina $a$ are guided by specific rules of contact and not representative of some baseline "random" contacts.

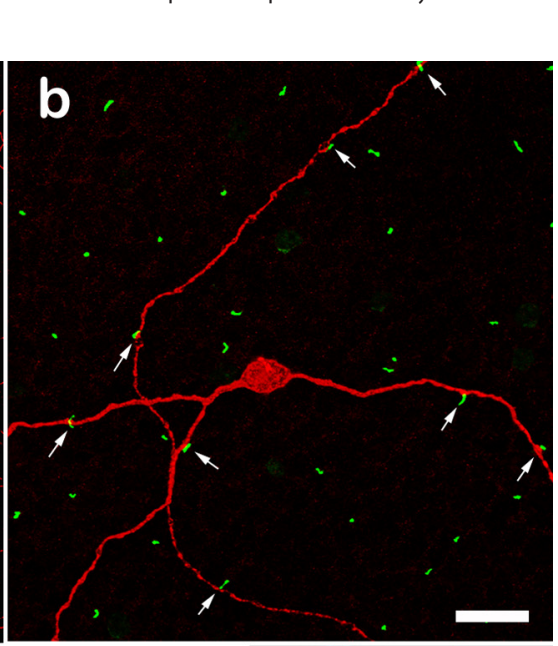

Figure 4. Millipore antibody MAB3101 stains ipRGC somas and processes. These can ramify in sublamina $a(\boldsymbol{a})$, sublamina $b$, or the green ipRGC dendrites was reduced to maximize contrast. Scale bars: $\boldsymbol{a}, 50 \mu \mathrm{m} ; \boldsymbol{b}, 20 \mu \mathrm{m} ; \boldsymbol{c}, 10 \mu \mathrm{m} ; \boldsymbol{d}, 1 \mu \mathrm{m}$. Numbers of optical sections of $0.3 \mu \mathrm{m}$ thickness are as follows: $\boldsymbol{a}, 10 ; \boldsymbol{b}, 2 ; \boldsymbol{c}, 14 ; \boldsymbol{d}, 1$.

\section{Discussion}

Cajal recognized that the laminar structure of the retina in general, and the IPL specifically, might lend itself to the division of processing by level of stratification. The subsequent discovery of the functional division of the IPL into ON and OFF layers (Famiglietti et al., 1977; Nelson et al., 1978) has remained one of the most fundamental and enduring tenets of retinal morphology. Cells whose processes terminate in sublamina $a$ depolarize at light offset, and those that terminate in sublamina $b$ depolarize to light onset.

DACs provide a challenge to this rule. Despite sending a few processes to more proximal strata, their ribbon inputs occur in the most distal region (Hokoc and Mariani, 1987). However, this amacrine cell depolarizes to light (Kolb et al. 1997; Zhang et al., 2007), and dopamine release is blocked in a variety of vertebrates (Boelen et al., 1998) by application of L-APB, which blocks the ON pathway (Slaughter and Miller, 1981). The source of this excitatory input has remained unidentified.

Similarly, possible sources of excitatory ON inputs to ipRGC types that ramify in sublamina a (type M1) are unidentified. Both morphological and physiological evidence suggest that diverse populations of ipRGCs exist that spike at light onset regardless of whether they ramify in the ON or OFF layers of the IPL (Dacey et al., 2005; Tu et al., 2005; Viney et al., 2007; Schmidt and Kofuji, 2009). The response of ipRGCs to light onset is a long-latency sustained depolarization with spiking, an $\mathrm{ON}$ response (Berson et al., 2002). These cells also receive more transient inputs from traditional photoreceptors via bipolar cells. The transient inputs 


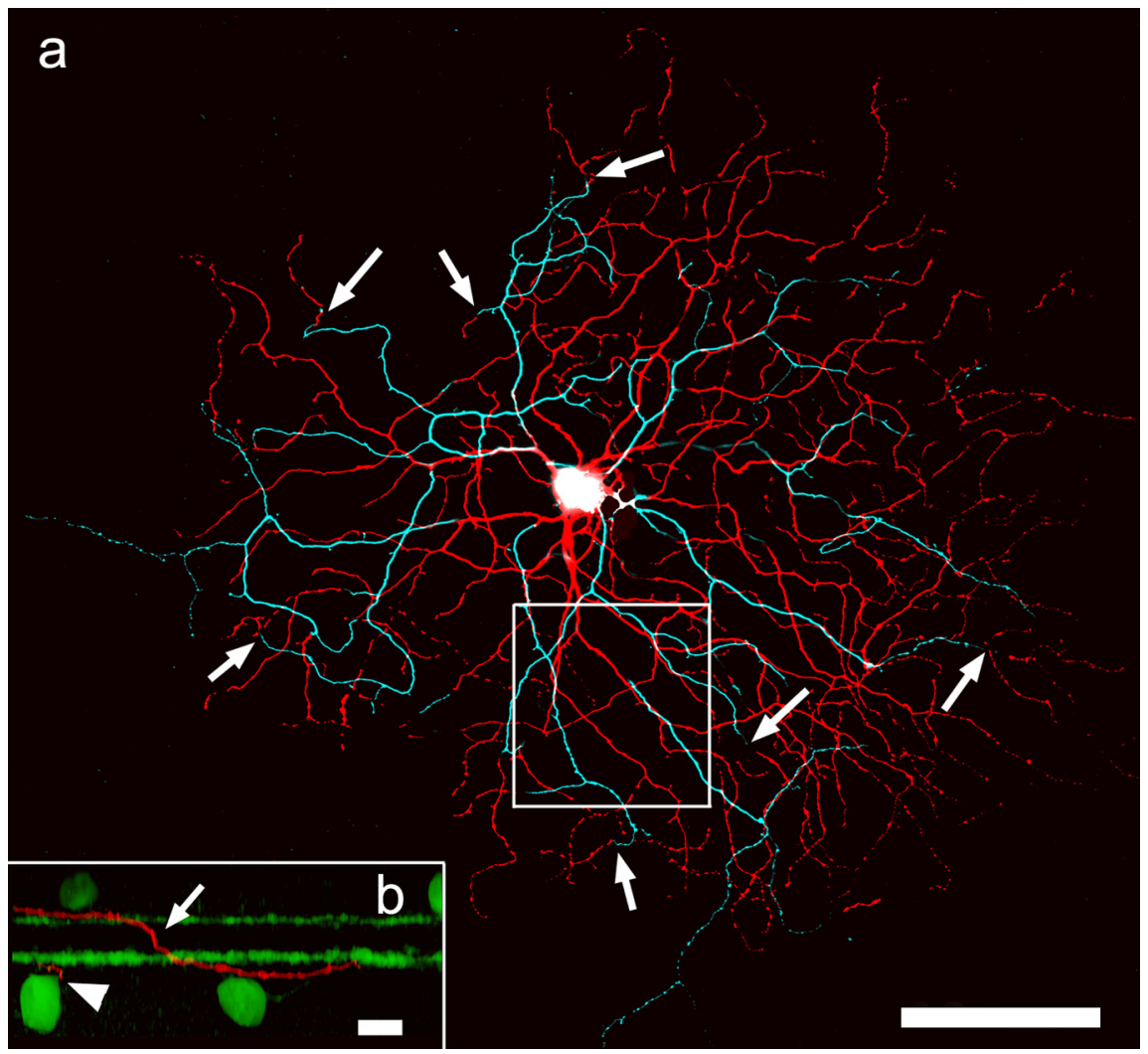

Figure 5. The bistratified diving ganglion cell is distinguished by arbors in both sublamina $a$ (cyan) and $b$ (red) and, most diagnostically, the return of the processes in sublamina $a$ to sublamina $b$ before their final termination (arrows). Some processes have their complete arbor in sublamina $b$, but many other processes in the periphery of the dendritic field appear disconnected at that level of focus. The disconnected processes in sublamina $b$ are connected to the dendritic arbor in sublamina $a$ at the point they descend back to sublamina $b$ (arrows). Inset, Rotation of optical sections into the $z$-axis shows how sharply dendrites in sublamina $a$ descend back to sublamina $b$ (arrow). The arrowhead marks the termination of a dendrite wholly stratified in sublamina $b$. The green bands are cholinergic processes stained with anti-ChAT for reference. The box indicates the area sampled in Figure 6. Scale bars: $100 \mu \mathrm{m}$; inset, $10 \mu \mathrm{m}$. All optical sections are $0.3 \mu \mathrm{m}$ thick. The main panel is a stack of $70 \times 0.3 \mu \mathrm{m}$ optical sections; the inset is a stack of $6 \times 0.3 \mu \mathrm{m}$ optical sections.

are blocked by L-APB (Dacey et al., 2005; Wong et al., 2007; Zhang et al., 2008), whereas the sustained response, which presumably represents the intrinsic melanopsin-driven photoresponse, is not blocked. Consistent with this, Jusuf et al. (2007) found putative ribbon synapses apposed to ipRGCs in both layers of primate retina, although Belenky et al. (2003) found bipolar cell inputs to ipRGCs only in the ON layer of mouse. Together, this set of results suggest that the axonal ribbons we have shown may function as excitatory cone inputs to $\mathrm{ON}$ responding neurons anomalously ramifying in sublamina $a$. Recently, Pickard et al. (2009) demonstrated light-onset-induced c-Fos expression in M1 ipRGCs even in melanopsin knock-out mice, i.e., ON inputs originating from traditional photoreceptors are found in these cells whose arbors are wholly contained in sublamina $a$. Our results provide morphological evidence for their "direct" model (Pickard et al., 2009, their Fig. 3).

Calbindin-positive bipolar cells synapse onto ON-responding cells in sublamina $a$ as well as sublamina $b$

This study provides extensive evidence for input from ON bipolar cells to three different postsynaptic targets ramifying in the OFF sublamina. We investigated possible synaptic contacts between the axons of the calbindin-immunoreactive ON bipolar cell (Massey and Mills, 1996) with spines and dendrites of three cell types whose processes branch in sublamina $a$ : (1) DACs, (2)
ipRGCs, which are intrinsically photosensitive via a melanopsin cascade (Berson et al., 2002; Güler et al., 2008), and (3) bistratified diving ganglion cells, named after their unusual and diagnostic branching pattern. In each case, the input was from axons of the calbindin-positive bipolar cell, ribbon synapses were present, and a postsynaptic marker (GluR4) was also found at these sites. We are unable with purely anatomical methods to demonstrate that these putative synapses are truly functional. However, these markers strongly suggest the presence of physiological synapses that can provide ON input despite being located in sublamina $a$.

There are three distinct physiological types of DACs in mouse retina (Zhang et al., 2007), but no morphological attributes could be distinguished that would allow us to discriminate possible subtypes in the rabbit retina. In our study, all DACs made contact with descending axons of calbindin-positive bipolar cells. Either the DACs in rabbit retina are a homogeneous population or all types are contacted by the calbindin-positive ON cone bipolar cell.

The functional stratification of the IPL Electron microscopy has demonstrated that many of the bipolar cell inputs to the DACs of cat and rabbit retina occur at monadic synapses (Hokoc and Mariani, 1987) rather than the normal dyadic arrangement in the IPL. This suggests that axonal synapses might not form full dyads. Because $99 \%$ of the ribbons contained in the axons of calbindin-positive bipolar cells were located in stratum 1 rather than lower in sublamina $a$, this might indicate a zone of ON input high in sublamina $a$ that could be distal to the OFF layer. Alternatively, there might be a small, overlapping region in which $\mathrm{ON}$ and OFF inputs are not exclusively segregated by stratification depth.

There are some limited precedents for these unusual findings. Electron micrographs showed ribbons in stratum 1 of primate blue cone bipolar cells (Calkins et al., 1998) and in two ON cone bipolar cell types in the cat (McGuire et al., 1984), and $\mathrm{Ca}^{2+}$ channels were sometimes found in axons of isolated rod bipolar cells (Berntson et al., 2003). Also, synapses have been reported between the somas of bipolar cells and amacrine cells in cat retina (Fisher, 1972), although the synapse was from the amacrine cell onto the bipolar cell, unlike the ribbon synapse we report.

\section{Function of en passant synapses}

Our results indicate violation of the canonical division of the IPL into OFF and $\mathrm{ON}$ sublaminae. Two questions that naturally arise are as follows: (1) why DACs, which depolarize to light onset, ramify predominantly in the OFF layers, and (2) why en passant synapses with apparent ON polarity occur in the OFF layer. The answers are not clear, but we note that, because DACs regulate outer retinal functions through paracrine release of dopamine in the IPL, it would be most efficient for them to be located as near these targets as practical. Given this location of the DACs, one 
possible reason why some ipRGCs ramify in sublamina a may be to provide input to the dopaminergic system (Zhang et al., 2008).

The number of synapses made by the calbindin-positive bipolar cell in sublamina $a$ is small compared with those in sublamina $b$. This raises the question of whether the visual function they perform in the OFF layer differs from that in the ON layer. Neither DACs nor ipRGCs provide fine spatial information and therefore do not require a strong, spatially localized input. With respect to en passant synapses from calbindin-positive bipolar cells onto the bistratified diving ganglion cell, it may be that EPSPs at the most distal processes of this ganglion cell might fail to propagate to the soma without reinforcing inputs that arise from the en passant synapses onto the arbor in sublamina $a$.

\section{Do other ON cone bipolar cells make synapses en passant?}

Only $\sim 26.3 \pm 2.0 \%$ of the dendritic spines of four bistratified diving ganglion cells were apposed to calbindin-positive bipolar cells (all of which contained ribbons at these junctures). The remaining 74\% of the spines were also almost all associated with ribbon synapses. This suggests that they are postsynaptic to $\mathrm{ON}$ cone bipolar cells of a different type. Input from OFF cone bipolar cells is excluded by the lack of spiking activity in L-APB-treated retinas.

It should also be noted that the low number $(6 \%)$ of calbindin-positive bipolar cell axons that contact ipRGCs is a reflection of the very sparse nature of ipRGC dendrites; when dendrites do occur, putative axonal synapses are commonplace (Fig. 4). Because we are unable to detect axonal synapses from unlabeled ON bipolar cells, these potentially could contribute additional excitatory input to the targets we have identified.

\section{ON cone bipolar cell inputs to DACs}

The number of inputs from descending axons of calbindinpositive bipolar cells to DACs can be calculated from data such as Figure $2 a$ to be $\sim 775$ ribbons $/ \mathrm{mm}^{2}$. The presence of GluR4 staining on DACs apposed to ribbons not contained in calbindin-positive bipolar cells suggests potential en passant synapses from other types of $\mathrm{ON}$ cone bipolar cells. Using size and density data for the type 1 catecholamine-accumulating cell (Tauchi et al., 1990), which closely resembles the DAC, we calculate a coverage factor for these cells of $\sim 8.8$. This suggests that each $\mathrm{DAC}$ receives $\sim 775 / 8.8=88$ axonal ribbon synapses from calbindin-positive bipolar cells alone. This is a substantial input that finally reveals both the long-uncertain source of ON excitation to DACs and how it arrives at a cell type that ramifies in the OFF layer.
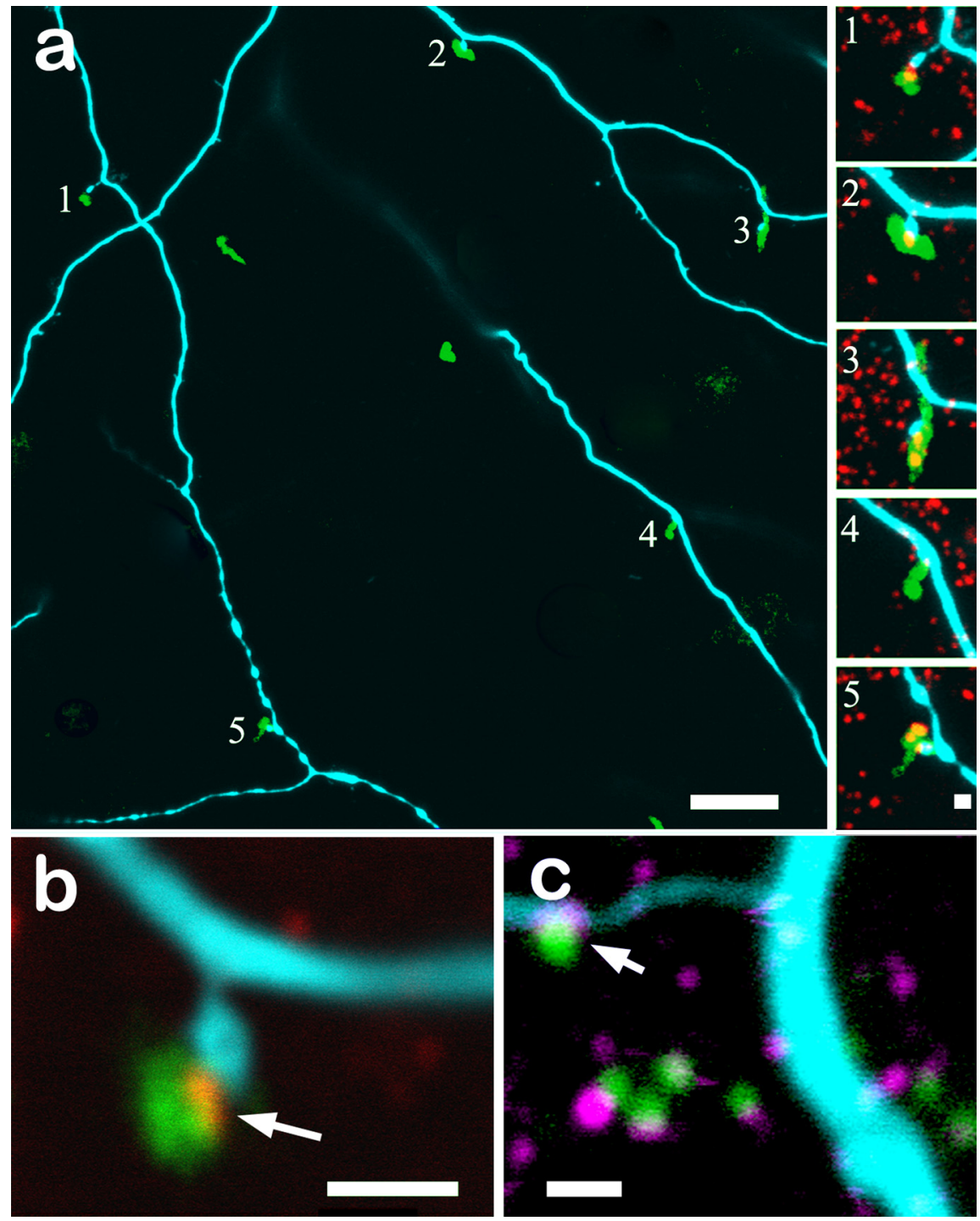

Figure 6. $\quad \boldsymbol{a}$, The dendritic arbor of the bistratified diving ganglion cell shown in Figure 5 contains numerous spines normally associated with synaptic activity in sublamina $a$ (cyan). These spines frequently contact the descending axons of calbindin-positive bipolar cells (green). All five spines in this field also contain RIBEYE immunoreactivity (red; insets $\boldsymbol{a} 1-\boldsymbol{a}$ ) in which the spine conjunction of the bistratified diving ganglion cell spine (blue) and the calbindin-positive bipolar cell axon (green). c, Contact points of the bistratified diving ganglion cell (cyan) with calbindin-positive bipolar cells axons (green) also contain glutamate receptors, as seen by immunostaining of the AMPA receptor GluR4 (magenta). Scale bars: $\boldsymbol{a}, 10 \mu \mathrm{m} ; \boldsymbol{a} \mathbf{1}-\boldsymbol{a} \mathbf{5}, \boldsymbol{b}, \boldsymbol{c}, 1 \mu \mathrm{m}$. Nine optical sections of $0.3 \mu \mathrm{m}$ thickness are stacked in $\boldsymbol{a}$. All other panels are single $0.3 \mu \mathrm{m}$ sections.

\section{References}

Amthor FR, Oyster CW, Takahashi ES (1984) Morphology of on-off directionselective ganglion cells in the rabbit retina. Brain Res 298:187-190.

Baver SB, Pickard GE, Sollars PJ, Pickard GE (2008) Two types of melanopsin retinal ganglion cell differentially innervate the hypothalamic suprachiasmatic nucleus and the olivary pretectal nucleus. Eur J Neurosci 27:1763-1770.

Belenky MA, Smeraski CA, Provencio I, Sollars PJ, Pickard GE (2003) Melanopsin retinal ganglion cells receive bipolar and amacrine cell synapses. J Comp Neurol 460:380-393.

Berntson A, Taylor WR, Morgans CW (2003) Molecular identity, synaptic localization, and physiology of calcium channels in retinal bipolar cells. J Neurosci Res 71:146-151.

Berson DM, Dunn FA, Takao M (2002) Phototransduction by retinal ganglion cells that set the circadian clock. Science 295:1070-1073.

Boelen MK, Boelen MG, Marshak DW (1998) Light-stimulated release of dopamine from the primate retina is blocked by 1-2-amino-4phosphonobutyric acid (APB). Vis Neurosci 15:97-103. 
Calkins DJ, Tsukamoto Y, Sterling P (1998) Microcircuitry and mosaic of a blue-yellow ganglion cell in the primate retina. J Neurosci 18:3373-3385.

Dacey DM, Liao HW, Peterson BB, Robinson FR, Smith VC, Pokorny J, Yau KW, Gamlin PD (2005) Melanopsin-expressing ganglion cells in primate retina signal colour and irradiance and project to the LGN. Nature 433:749-754.

DeVries SH (2000) Bipolar cells use kainate and AMPA receptors to filter visual information into separate channels. Neuron 28:847-856.

Famiglietti EV Jr, Kaneko A, Tachibana M (1977) Neuronal architecture of on and off pathways to ganglion cells in carp retina. Science 198:1267-1269.

Fisher SK (1972) A somato-somatic synapse between amacrine and bipolar cells in the cat retina. Brain Res 43:587-590.

Güler AD, Ecker JL, Lall GS, Haq S, Altimus CM, Liao HW, Barnard AR, Cahill H, Badea TC, Zhao H, Hankins MW, Berson DM, Lucas RJ, Yau KW, Hattar S (2008) Melanopsin cells are the principal conduits for rod-cone input to non-image-forming vision. Nature 453:102-105.

Hattar S, Liao HW, Takao M, Berson DM, Yau KW (2002) Melanopsincontaining retinal ganglion cells: architecture, projections, and intrinsic photosensitivity. Science 295:1065-1070.

Hokoc JN, Mariani AP (1987) Tyrosine hydroxylase immunoreactivity in the rhesus monkey retina reveals synapses from bipolar cells to DACs. J Neurosci 7:2785-2793.

Hoshi H, Mills SL (2009) Components and properties of the G3 ganglion cell circuit in the rabbit retina. J Comp Neurol 513:69-82.

Johnson J, Sherry DM, Liu X, Fremeau RT Jr, Seal RP, Edwards RH, Copenhagen DR (2004) Vesicular glutamate transporter 3 expression identifies glutamatergic amacrine cells in the rodent retina. J Comp Neurol 477:386-398.

Jusuf PR, Lee SC, Hannibal J, Grünert U (2007) Characterization and synaptic connectivity of melanopsin-containing ganglion cells in the primate retina. Eur J Neurosci 26:2906-2921.

Kolb H, Netzer E, Ammermüller J (1997) Neural circuitry and light responses of the dopamine amacrine cell of the turtle retina. Mol Vis 3:6.

Marc RE, Jones BW (2002) Molecular phenotyping of retinal ganglion cells. J Neurosci 22:413-427.

Marshak DW (2001) Synaptic inputs to dopaminergic neurons in mammalian retinas. Prog Brain Res 131:83-91.

Massey SC, Mills SL (1996) A calbindin-immunoreactive bipolar cell type in the rabbit retina. J Comp Neurol 366:15-33.

McGuire BA, Stevens JK, Sterling P (1984) Microcircuitry of bipolar cells in cat retina. J Neurosci 4:2920-2938.

Nelson R, Famiglietti EV Jr, Kolb H (1978) Intracellular staining reveals different levels of stratification for on- and off-center ganglion cells in cat retina. J Neurophysiol 41:472-483.

Nomura A, Shigemoto R, Nakamura Y, Okamoto N, Mizuno N, Nakanishi S (1994) Developmentally regulated postsynaptic localization of a metabotropic glutamate receptor in rat rod bipolar cells. Cell 77:361-369.

Pickard GE, Baver SB, Ogilvie MD, Sollars PJ (2009) Light-induced Fos Expression in intrinsically photosensitive retinal ganglion cells in melanopsin knockout (Opn4-/-) mice. PLOS One 4:e4984.

Rockhill RL, Daly FJ, MacNeil MA, Brown SP, Masland RH (2002) The diversity of ganglion cells in a mammalian retina. J Neurosci 22:3831-3843.

Roska B, Werblin F (2003) Rapid global shifts in natural scenes block spiking in specific ganglion cell types. Nat Neurosci 6:600-608.

Roska B, Molnar A, Werblin FS (2006) Parallel processing in retinal ganglion cells: how integration of space-time patterns of excitation and inhibition form the spiking output. J Neurophysiol 95:3810-3822.

Schmidt TM, Kofuji P (2009) Functional and morphological differences among intrinsically photosensitive retinal ganglion cells. J Neurosci 29:476-482.

Slaughter MM, Miller RF (1981) 2-amino-4-phosphonobutyric acid: a new pharmacological tool for retina research. Science 211:182-185.

Tauchi M, Madigan NK, Masland RH (1990) Shapes and distributions of the catecholamine-accumulating neurons in the rabbit retina. J Comp Neurol 293:178-189.

Tu DC, Zhang D, Demas J, Slutsky EB, Provencio I, Holy TE, Van Gelder RN (2005) Physiologic diversity and development of intrinsically photosensitive retinal ganglion cells. Neuron 48:987-999.

Viney TJ, Balint K, Hillier D, Siegert S, Boldogkoi Z, Enquist LW, Meister M, Cepko CL, Roska B (2007) Local retinal circuits of melanopsincontaining ganglion cells identified by transsynaptic viral tracing. Curr Biol 17:981-988.

Wässle H (2004) Parallel processing in the retina. Nat Rev Neurosci 5:747-757.

Witkovsky P (2004) Dopamine and retinal function. Doc Ophthalmol 108:17-40.

Wong KY, Dunn FA, Graham DM, Berson DM (2007) Synaptic influences on rat ganglion-cell photoreceptors. J Physiol 582:279-296.

Zhang DQ, Zhou TR, McMahon DG (2007) Functional heterogeneity of retinal dopaminergic neurons underlying their multiple roles in vision. J Neurosci 27:692-699.

Zhang DQ, Wong KY, Sollars PJ, Berson DM, Pickard GE, McMahon DG (2008) Intraretinal signaling by ganglion cell photoreceptors to dopaminergic amacrine neurons. Proc Natl Acad Sci U S A 105:14181-14186. 